\title{
Multi-Connectivity based Uplink/Downlink decoupled Energy Efficient User Association in 5G Heterogenous CRAN
}

\author{
Merve Saimler and Sinem Coleri, Senior Member, IEEE
}

\begin{abstract}
Multi-connectivity $(M C)$ is proposed in FifthGeneration mobile communications systems $(5 G)$ to mitigate the deterioration of Quality-of-Service owing to line-of-sight blockage and lack of communication resources. The main idea of MC is to associate single user equipment with multiple network layers and multiple radio access technologies, simultaneously. In previous studies, MC is demonstrated to increase capacity, provide high reliability and decrease outage probability. However, for the first time in literature, in this paper, we incorporate $\mathrm{MC}$ into the optimization of the total power consumption in 5G Heterogeneous Cloud Radio Access Networks. Upon formulation of the problem as a binary integer linear programming problem, proving its NP-hardness, a heuristic algorithm is proposed consisting of linear programming relaxation and rounding and generalized assignment problem heuristic, which takes the outputs of the linear programming relaxation and rounding as inputs and checks if it further minimizes the power consumption. We analyze power consumption, time complexity and achievable rate of the proposed algorithm and verify its superiority over existing methods by simulations.
\end{abstract}

\section{INTRODUCTION}

5G Heterogeneous Cloud Radio Access Network $(H C R A N)$ consists of Macro Base Stations $(M B S)$ s, which operate at conventional cellular frequencies and provide coverage, and Small Base Stations $(S B S)$ s, which operate at millimeter wave ( $m m W$ ave) frequencies and are densely deployed in hot spots to provide high data rates to user equipments $(U E)$ s. Signal processing in MBS and SBS is coordinated by Baseband Unit Cloud ( $B B U-C l o u d)$, which provides network controllability and flexibility [1]. Since received signal strength at mmWave is extremely susceptible to blockages due to short wavelengths, Multiconnectivity $(M C)$ is proposed to mitigate the resulting Quality-of-Service $(Q \circ S)$ degradation by associating a single UE with multiple network layers and multiple radio access technologies (RAT)s, simultaneously [2].

MC has been studied with the objectives increasing data rate, providing higher reliability and decreasing outage probability. Extremely high data rates are achieved by multiple $5 \mathrm{G}$ base stations $(B S)$ s [3]; highly reliable transmission is facilitated by combining multiple links[4]; and outage probability is decreased [5]. However, the usage of MC increases the power consumption, since in dense networks large number of BSs are activated in an uncontrolled manner to satisfy the aforementioned research objectives. None of the previous studies incorporates MC into the minimization of the system power consumption.

The minimization of the power consumption problem has been extensively studied for the case, where each UE associates with only one BS. The system power consumption is minimized by planning the activation/deactivation periods of SBSs in a controlled manner while satisfying the QoS constraints of UEs, which are minimum rate requirement [6], [7], outage probability [8] and latency of data transmission between UE and BSs [9]. However, methods implemented for single BS-UE associations cannot be applied to $\mathrm{MC}$ scenarios, since MC requires a centralized system with shared MAC layer to provide instant information about the timing of any topology changes to all UE-BS pairs in the network and facilitates rapid connection shifting if the connection is blocked unexpectedly.

This paper proposes a MC based Uplink $(U L) /$ Downlink $(D L)$ Decoupled energy efficient UE association in HCRAN for the first time in the literature. The proposed framework encompasses realistic power consumption models, including both signaling overhead and transmissions within the BBUCloud, which is key in the analysis of the advantage of the usage of multiple BSs. Moreover, UL/DL decoupled UEBS association and transmission with effective rate are used to further improve the performance through higher system flexibility.

\section{System Model AND ASSUMPTIONS}

We consider a HCRAN architecture, consisting of a MBS with $N_{R F, M}$ RF chains, which is operating at conventional cellular frequencies and $K$ SBSs with $N_{R F, S}$ RF chains, which are operating at mmWave frequencies in the service area of MBS. Additionally, $N_{b}$ mobile UEs exist in the service area of MBS and have a single antenna. UEs can get service from more than one BS, which are operating at conventional cellular frequencies and mmWave frequencies and can cooperate to serve UEs both in DL and UL, therefore, UE demand is splittable. The signal processing is managed by the BBU-Cloud, where the BSs are assumed to be connected to the BBU-Cloud through fronthaul $(F H)$ links, that have limited capacity, $C_{f h}$

$\alpha_{M j}^{t}$ and $\alpha_{S j}^{t}$ represent the attenuation levels at time $t$ in DL from MBS and SBS $S$ to UE $j$, respectively [10]. $\theta_{M j}^{t}$ and $\theta_{S j}^{t}$ indicate the attenuation levels in UL at time $t$ from $\mathrm{UE} j$ to MBS and SBS, respectively [10].

The power assignment at the UE $\mathrm{j}$ at time $t$ is executed to overcome $\epsilon$ fraction of the path loss with respect to a reference received power per resource block at $0 \mathrm{~dB}$ path loss, $P_{0}$, below $P_{\max }$ and is formulated as $P_{t, U E}^{t}=$ $\min \left\{P_{\max }, P_{0}+\epsilon * \alpha_{\gamma, j}^{t}\right\}$, where $\gamma$ is $\mathrm{S}$ or $\mathrm{M}$ [11].

The effective achievable rate at time t for $N_{c}$ cooperating BSs in DL is formulated as $\tilde{R}_{d, \gamma, j}^{t}=\frac{1}{N_{c}}\left(\sum_{i=1}^{N_{c}} R_{d, \gamma, j, i}^{t} * \phi\right)$ [12], where $\gamma$ is $\mathrm{S}$ or $\mathrm{M}$, and $R_{d, S, j, i}^{t}$ and $R_{d, M, j, i}^{t}$ are the achievable rates at time $\mathrm{t}$ in DL at UE $j$ by the $i^{\text {th }}$ cooperating SBS $S$ and MBS, respectively. Achievable rate is formulated as $B_{\gamma, i} * \log \left(1+\frac{P_{t, \gamma, i} * \alpha_{\gamma, j, i}^{t}}{\left|N_{R F, \gamma, i}\right| * N_{0} * B_{\gamma, i}+P_{j, I d l}^{t}}\right)$, where $B_{S, i}$ and $B_{M, i}$ denote bandwidth required for transmission 
for the $i^{\text {th }}$ cooperating SBS $S$ and MBS, respectively [10], $N_{R F, S, i}$ and $N_{R F, M, i}$ are numbers of RF chains and are equivalent to the number of UEs at maximum that can be provided service by the $i^{\text {th }}$ cooperating SBS $S$ and MBS, respectively [13]. $P_{t, S, i}$ or $P_{t, M, i}$ demonstrate the transmit power of $i^{\text {th }}$ cooperating SBS $S$ and MBS, respectively, $N_{0}$ is the power spectral density of the noise and $P_{j, I d l}^{t}$ represents the power of interference at time $\mathrm{t}$ in DL. $\phi$ is the value indicating how much $R_{d, \gamma, j, i}^{t}$ degrades due to orthogonal and pre-coded pilots used by UEs to estimate and feedback channel matrix and for equalization and decoding, respectively. It is formulated as $\left(1-\rho * 3 * N_{c}\right)-\delta$, where $\rho$ represents the average pilot density in time and frequency and $\delta$ denotes the average control overhead [12].

The effective achievable rate at time $\mathrm{t}$ in $\mathrm{UL}$ for $N_{c}$ cooperating BSs reduces on average to $\tilde{R}_{u, \gamma, j}^{t}=$ $\frac{1}{N_{c}}\left(\sum_{i=1}^{N_{c}} R_{u, \gamma, j, i}^{t} * \Theta\right)$ [12], where $\gamma$ is $\mathrm{S}$ or $\mathbf{M}$, and $R_{u, S, j, i}^{t}$ and $R_{u, M, j, i}^{t}$ are the achievable rates at time $\mathrm{t}$ in UL at UE $j$ by the $i^{\text {th }}$ cooperating SBS $S$ and MBS, respectively. Achievable rate is formulated as $B_{\gamma, i} *$ $\log \left(1+\frac{P_{t, j, i}^{t} * \theta_{\gamma, j, i}^{t}}{N_{0} * B_{\gamma, i}+P_{j, \text { Iul }}^{t}}\right)$, where $\gamma$ is S or M, $P_{t, j, i}$ represents the transmit power of UE $j$ to the $i^{\text {th }}$ cooperating SBS $S$ or MBS; and $P_{j, I u l}^{t}$ represents the power of interference at time $\mathrm{t}$ in UL at UE $j$ [10]. $\Theta$ is an expression of how much $R_{u, \gamma, j, i}^{t}$ degrades due to pilot signaling and CSI feedback rate, which are both scaled with the total number of UL transmit antennas within a cluster linearly. The amount of degradation in UL rate is formulated as $\left(1-\rho * N_{c}\right)-\left(2 * N_{c} * \rho * q\right)$, where $q$ represents the feedback bits that are used to calculate CSI for QoS [12].

$Q_{d, j}^{t}$ and $Q_{u, j}^{t}$ are data rates requested by $\mathrm{UE} j$ at time $t$ in DL and UL, respectively.

MBS power consumption at time $t$ encompasses static power consumption, $P_{S t}$ and dynamic power consumption at time $t, P_{d y n}^{t}$ [14]. $P_{S}$ is the power consumption of SBS $S$ for all traffic load [14]. $P_{u, f h l}^{t}$ and $P_{d, f h l}^{t}$ represent the power consumption values of FH link at time t, in UL and DL, respectively [15]. $P_{u, j}^{t}$ is the power consumption of a UE $j$ at time t [16]. $P_{S P}$ is the power consumption of baseband digital signal processing [12].

\section{OptimizATION OF MC BASED UE ASSOCIATION}

The optimization of MC based UL/DL decoupled UE association scheme with the objective of minimizing the power consumption by incorporating static and dynamic power consumption of MBS, power consumption of $\mathrm{FH}$ links and UEs in UL, activation/deactivation operation of SBSs and signal processing is formulated as:

$$
\begin{array}{ll}
\min & \sum_{j=1}^{N_{b}}\left(\frac{Q_{d, j}^{t}}{R_{d, M j}^{t}}\right) * P_{d y n} * x_{M j}^{t}+\sum_{S=1}^{K} P_{S} * T_{S}^{t}+P_{f h} * \sum_{j=1}^{N_{b}} Q_{d, j}^{t} * x_{M j}^{t}+ \\
& \sum_{S=1}^{K} P_{f h} *\left(\sum_{j=1}^{N_{b}} Q_{d, j}^{t} * x_{S j}^{t}\right)+P_{S P}+w *\left(\sum_{S=1}^{K} P_{f h} *\left(\sum_{j=1}^{N_{b}} Q_{u, j}^{t} * y_{S j}^{t}\right)\right)+ \\
& \left(\sum_{j=1}^{N_{b}}\left(\left(P_{u, j}^{t}+P_{d y n}\right) * \frac{Q_{u, j}^{t}}{R_{u, M j}^{t}}\right) * y_{M j}^{t}+\sum_{j=1}^{N_{b}} P_{u, j}^{t} \sum_{s=1}^{K} \frac{Q_{u, j}^{t}}{R_{u, S j}^{t}} * y_{S j}^{t}\right)+P_{s t} \\
\text { s.t. } \quad & \frac{1}{N_{C}} *\left(\phi * R_{d, M j}^{t} * H_{M j}^{t}+\sum_{S=1}^{K} \phi * R_{d, S j}^{t} * C_{S j}^{t}\right) \geq Q_{d, j}^{t},
\end{array}
$$

$$
\begin{aligned}
& j \in\left\{1, N_{b}\right\}, t \in\{1, T\} \\
& \frac{1}{N_{c}} *\left(\Theta * R_{u, M j}^{t} * G_{M j}^{t}+\sum_{S=1}^{K} \Theta * R_{u, S j}^{t} * Z_{S j}^{t}\right) \geq Q_{u, j}^{t}, \\
& j \in\left\{1, N_{b}\right\}, t \in\{1, T\} \\
& \sum_{j=1}^{N_{b}} \frac{Q_{d, j}^{t}}{R_{d, M j}^{t}} * x_{M j}^{t}+\sum_{j=1}^{N_{b}} \frac{Q_{u, j}^{t}}{R_{u, M j}^{t}} * y_{M j}^{t} \leq N_{R F, M}, t \in\{1, T\} \\
& \sum_{j=1}^{N_{b}} \frac{Q_{d, j}^{t}}{R_{d, S j}^{t}} * x_{S j}^{t}+\sum_{j=1}^{N_{b}} \frac{Q_{u, j}^{t}}{R_{u, S j}^{t}} * y_{S j}^{t} \leq N_{R F, S}, S \in\{1, K\}, t \in\{1, T\} \\
& \sum_{j=1}^{N_{b}} Q_{d, j}^{t} * x_{S j}^{t}+\sum_{j=1}^{N_{b}} Q_{u, j}^{t} * y_{S j}^{t} \leq C_{f h}, S \in\{1, K\}, t \in\{1, T\} \\
& \sum_{j=1}^{N_{b}} Q_{d, j}^{t} * x_{M j}^{t}+\sum_{j=1}^{N_{b}} Q_{u, j}^{t} * y_{M j}^{t} \leq C_{f h}, S \in\{1, K\}, t \in\{1, T\} \\
& x_{S j}^{t} \leq T_{S}^{t}, j \in\left\{1, N_{b}\right\}, S \in\{1, K\}, t \in\{1, T\} \\
& y_{S j}^{t} \leq T_{S}^{t}, j \in\left\{1, N_{b}\right\}, S \in\{1, K\}, t \in\{1, T\} \\
& x_{S j}^{t} \leq C_{S j}^{t}, j \in\left\{1, N_{b}\right\}, S \in\{1, K\}, t \in\{1, T\} \\
& y_{S j}^{t} \leq Z_{S j}^{t}, j \in\left\{1, N_{b}\right\}, S \in\{1, K\}, t \in\{1, T\} \\
& x_{M j}^{t} \leq H_{M j}^{t}, j \in\left\{1, N_{b}\right\}, t \in\{1, T\} \\
& y_{M j}^{t} \leq G_{M j}^{t}, j \in\left\{1, N_{b}\right\}, t \in\{1, T\} \\
& H_{M j}^{t}+\sum_{S=1}^{K} C_{S j}^{t} \leq N_{c}, j \in\left\{1, N_{b}\right\}, t \in\{1, T\} \\
& G_{M j}^{t}+\sum_{S=1}^{K} z_{S j}^{t} \leq N_{c}, j \in\left\{1, N_{b}\right\}, t \in\{1, T\} \\
& \text { Vs } T_{S}^{t}, C_{S j}^{t}, z_{S j}^{t}, H_{M j}^{t}, G_{M j}^{t} x_{M j}^{t}, x_{S j}^{t}, y_{M j}^{t}, y_{S j}^{t} \in\{0,1\} \text {, }
\end{aligned}
$$

where $K$ is the number of SBSs, $N_{b}$ is the number of UEs and $T$ is the number of time-slots. The decision variables are $T_{S}^{t}$, that is a binary variable with value 1 in case SBS $S$ is activated when $U E$ is assigned with the corresponding SBS $S$ at time t in DL and UL and 0 otherwise; $C_{S j}^{t}$ and $H_{M j}^{t}$, that are binary variables with values 1 in case SBS $S$ and MBS cooperates with other BSs to serve UE $j$ at time $\mathrm{t}$ in DL, respectively, and 0 otherwise; $Z_{S j}^{t}$ and $G_{M j}^{t}$, that are binary variables with values 1 in case SBS $S$ and MBS cooperates with other BSs to serve UE $j$ at time $\mathrm{t}$ in UL, respectively, and 0 otherwise; $x_{S j}^{t}$ and $x_{M j}^{t}$, that are binary variables with values 1 when UE $j$ is assigned to SBS $S$ and MBS at time $\mathrm{t}$ in DL, respectively, and 0 otherwise; $y_{S j}^{t}$ and $y_{M j}^{t}$, that are binary variables with values 1 when UE $j$ is assigned to SBS $S$ and MBS at time $\mathrm{t}$ in UL, respectively, and 0 otherwise.

Constraints (1a) and (1b) represent that the data rate requested by UE $j$ at time $\mathrm{t}$ in DL and $\mathrm{UL}$ are provided by one BS or more than one BS, respectively. Constraints (1c) and (1d) indicate that the numbers of RF chains are equivalent to the number of UEs at maximum that can be provided service by the MBS and SBS $S$, respectively, at time t. Constraints (1e) and (1f) demonstrate that the data flow of the UEs on FH link at time t both in DL and UL cannot be greater than the total FH link capacity. Constraints (1g) and Constraints (1h) imply that if any UE associates with SBS $S$ at time t in DL and UL, SBS $S$ is activated. Additionally, constraints (1i) and (1j) ensure that SBS $S$ can cooperate with other BSs to provide service to UE $j$ at time t in DL and UL, respectively. Constraints (1k) and (11) ensure that MBS can cooperate with other BSs to provide service to UE $j$ at time t in DL and UL, respectively. Constraints $(1 \mathrm{~m})$ and $(1 \mathrm{n})$ represent that number of BSs that cooperate to serve UE $j$ at time $\mathrm{t}$ in DL and UL is at most $N_{c}$. 
Theorem 1. The optimization problem (1) is NP-hard.

Proof. We reduce our optimization problem to NP-Hard uncapacitated k-facility median problem.

Let us define a problem instance, where the number of cooperating BSs, $N_{c}$, is equal to one. Then, only one BS becomes active to serve a UE in UL and DL, which constrains variables $H_{M j}^{t}, G_{M j}^{t}, C_{S j}^{t}$ and $B_{S j}^{t}$ to be binary and make the constraints (1i)-(11) redundant. The problem reduces to the cost minimization problem in [17], in which $\mathrm{k}$ facilities are activated, where $\mathrm{k}$ is also the number of active BSs in our problem instance, and clients are associated with active facilities, such that demands of clients and capacity of facilities are satisfied, while minimizing the sum of transportation and facility location costs. It is proven to be NP-Hard, as a result of the reduction of the problem to NPHard uncapacitated k-facility median problem.

\section{A. MC based UE Association Algorithm}

MC based UE Association Algorithm ( $M C U A A)$, is proposed to solve the optimization problem (1) in polynomial time. The objective of MCUAA is to improve the initial solution, which is attained by using LP Relaxation-Rounding $(L P R-R)$ and Generalized Assignment Problem (GAP) heuristic, which takes the output of LPR-R as input and checks if it is possible to decrease the power consumption further [18]. In previously proposed Variable Neighborhood Search $(V N S)$ based heuristic algorithm to solve Capacitated k-facility location problem $(C K F L P)$ [17], the UE$\mathrm{BS}$ association and activation/deactivation of BSs is encoded as a random binary string, where each bit in the string may correspond to one potential activated/deactivated BS or assigned UE-BS pair. The current solution is improved by selecting a pre-defined UE-BS association method, changing the current UE-BS association with respect to pre-defined association method and then applying local search. On the contrary, in MCUAA, $L P R-R$ provides input, where the input consists of inactive BSs, unassigned UEs and assigned UE-BS pairs, to the following GAP heuristic to solve manyto-many assignment problem. The complexity is reduced by using $L P R-R$ and GAP heuristic, fast and extensive search for the minimum is enabled and MCUAA provides results, which are close to optimal.

MCUAA is demonstrated in Fig. 1. The flowchart begins with the introduction of the counter at time $\mathrm{t}\left(C N^{t}\right), C_{T H}$, the Local Value at time $\mathrm{t}\left(L V^{t}\right)$, the Main Value at time $\mathrm{t}\left(M V^{t}\right)$ and the Reference Value at time $\mathrm{t}\left(R V^{t}\right) . C N^{t}$ considers the number of times the heuristic runs with the value $L V^{t}$ less than $R V^{t}$ at time t, $C_{T H}$ is the number of count processes at maximum, $L V^{t}$ and $M V^{t}$ are equal to the values of power consumption at time $t$, attained from GAP heuristic and MCUAA, respectively, and $R V^{t}$ is obtained by the summation of power consumption values attained by $L P R-R$, and association of unassigned UEs with any random active $\mathrm{BS}$ at time $\mathrm{t}$.

Problem (1), binary integer linear programming (BILP), is resolved with the help of $L P R-R$ and the attained power consumption value is introduced as $R V^{t}$. Next, the solution demonstrates sets consisting of inactive BSs, unassigned UEs and assigned UE-BS pairs. The solution of $L P R-R$ is received by the GAP heuristic, where the main objective is to associate unassigned UEs with active BSs. In case where the active BSs cannot serve the unassigned UEs, an inactive $\mathrm{BS}$ is activated randomly. Otherwise, UEs, which are unassigned, are associated with the active BSs and $L V^{t}$ becomes equal to the power consumption value of assigned UE-BS pairs. Providing that the solution of GAP heuristic has less value than $R V^{t}$, an active BS is deactivated by MCUAA randomly, the UEs associated with the deactivated BS get unassigned and GAP heuristic is employed to examine, if it is possible to decrease the power consumption further by deactivating an active $\mathrm{BS}$, considering $C N^{t}$ has a lower value than $C_{T H}$. Conversely, in case when GAP heuristic cannot enable reduction in power consumption, MCUAA activates a BS randomly and examines if it is possible to decrease power consumption by activating inactive BSs, considering $C N^{t}$ has a lower value than $C_{T H}$ at time t.

Since a BS is selected from a set of active BSs, randomly, finding minimum power consuming BSs from the BS set, which consists of $m \mathrm{BS}$, is completed in $\mathcal{O}(\log m)$ time. Each of $n$ UEs is assigned to the active BSs, individually, where the association is completed in $\mathcal{O}(n)$ time. As a result, MCUAA is completed in $\mathcal{O}(n \log m)$ time.

Main functions of MCUAA are stated as follows:

1) Formation of Feasible Packings: Problem (1) is resolved with the help of $L P R-R$ and the solution demonstrates sets consisting of inactive BSs, unassigned UEs and assigned UE-BS pairs. These sets are obtained as follows:

After applying $L P R-R$, more than $N_{c}$ BSs cooperate to serve UEs. For each UE, we calculate the minimum power consuming $N_{c}$ BSs. The decision variable of the selected UE-BSs pair is set to 1 and the rest are set to zero.

After applying $L P R-R$, none of the UEs are associated with any BS and BSs are inactive. The decision variables are equal to zero.

After applying $L P R-R$, each UE is getting a service from an active BS or from a cooperating set of BSs. The decision variables of the corresponding association pairs are set to one and the rest is set to zero.

2) Heuristic for GAP: The solution of $L P R-R$, which demonstrates sets consisting of inactive BSs, unassigned UEs and assigned UE-BS pairs, is received by the GAP heuristic, where the main objective is to associate unassigned UEs with active BSs. GAP heuristic is employed to examine, if it is possible to decrease the power consumption further by deactivating an active BS.

The GAP heuristic functions as the following: Desirability measures $c_{s c j}^{t}, c_{m c j}^{t} c_{S j}^{t}$ and $c_{M j}^{t}$, at time $\mathrm{t}$ are introduced, which are assumed to be the values of power consumption of associating UE $j$ with cooperating set of SBSs, cooperating set of BSs including MBS, SBS $S$ and MBS, respectively. First, all unassigned UEs are considered and the corresponding $\mathrm{BS}$ is determined based on the maximum value of the difference between two smallest values of $c_{S j}^{t}$ and $c_{S c j}^{t}$ or 
$c_{M j}^{t}$ and $c_{M c j}^{t}$ values. The BS is then assigned to the UE $\mathrm{j}$ for which $c_{S j}^{t}, c_{S c j}^{t}, c_{M j}^{t}$ or $c_{M c j}^{t}$, is a minimum. The assignment is done for all unassigned UEs, iteratively. Next, current assignment scheme is improved by shifting, which considers each BS or cooperating BSs and re-assigns it to the UE $\mathrm{j}$ with minimum $c_{S j}^{t}, c_{S c j}^{t}, c_{M j}^{t}$ or $c_{M c j}^{t}$.

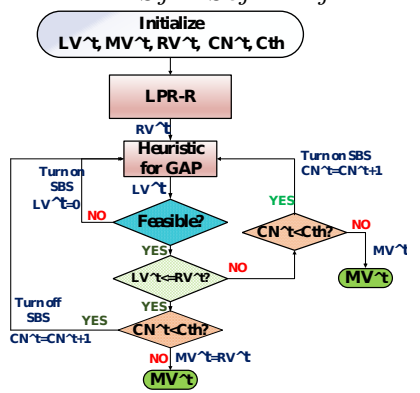

Fig. 1: MC based UE Association

IV. Performance Evaluation

The objective of the Section IV is to assess MCUAA compared to VNS-based heuristic algorithm, which is proposed for CKFLP [17]. VNS-based heuristic starts with an initial solution, which consists of inactive BSs, unassigned UEs and assigned UE-BS pairs. Then, by using one of the three neighborhood methods, which are active BS swapping, activating SBSs and deactivating SBSs, randomly, initial solution is improved and new assigned UE-BS pairs are obtained as output. The output of the selected neighborhood method is used as the input to the local search. Last, local search is used to search for the minimum power consuming solution in the corresponding neighborhood method and searches for the neighborhood method until the first improvement is found.

Simulation results are obtained by using IBM CPLEX Optimization Studio with MathWorks MATLAB and by averaging 10000 random configurations. Within the radius of $50 \mathrm{~m}$ to $700 \mathrm{~m}, 40$ SBSs are randomly and uniformly located with the minimum distance of 10 meters among each other and 100 UEs are deployed randomly and uniformly around BSs, which form hot spots, with velocities distributed between 0.1 $\mathrm{m} / \mathrm{s}$ and $0.5 \mathrm{~m} / \mathrm{s}$ randomly and uniformly. The links are assumed to be in Line-of-Sight $(L o S)$ or in Non-Line-Of-Sight $(N L o S)$ or in outage and the state changes with distance and mmWave-related environmental parameters [19]. The attenuation levels are stated using the path loss model and formulated as $\psi_{\gamma, j}^{t}=P L\left(d_{0}\right)^{t}+10 * \eta_{j}^{t} * \log _{10}\left(\frac{d_{\gamma, j}^{t}}{d_{0}}\right)+X_{\sigma_{j}}^{t}$, where $\gamma$ is $\mathbf{S}$ or $\mathrm{M}, P L\left(d_{0}\right)^{t}$ is the free space path loss in $\mathrm{dB}$ at a reference distance $d_{0}, \eta_{j}^{t}$ is the path loss exponent, $d_{\gamma, j}^{t}$ is the distance between $\mathrm{BS} \gamma$ and UE $j$, and $X_{\sigma_{j}}^{t}$ is a zero mean Gaussian random variable with $\left(\sigma_{j}^{t}\right)^{2}$ variance. The parameters for the mmWave path loss model at time $\mathrm{t}$ are stated in [19] and the rest are as follows: $B_{M}=20 \mathrm{MHz}$ and $B_{S}=800 \mathrm{MHz}, N_{0}=-134 \mathrm{dBm} / \mathrm{MHz}, C_{f h}=4.45 \mathrm{Gbps}$, $N_{R F, M}=8, N_{R F, S}=4, P_{f h}=0.1 \mathrm{~W} / \mathrm{Mbps}, \rho=8 / 168, \delta=3 / 14$, $q=8, P_{d y n}=768.9 P_{S}=12.1 \mathrm{~W} P_{S t}=605 \mathrm{~W}[14]$ and $Q_{d, j}^{t}$ and $Q_{u, j}^{t}$ are uniformly distributed between [0.15,2] Gbps and $[0.2,0.5]$ Gbps, respectively.

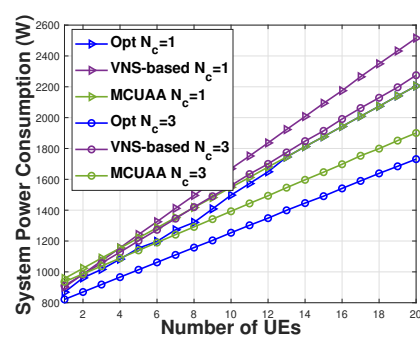

(a) number of the UEs

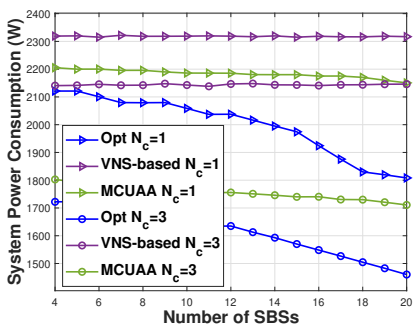

(b) number of the SBSs
Fig. 2: System Power Consumption

Fig. 2a shows the power consumption comparison results obtained for increasing number of the cooperating BSs and UEs. As the number of the UEs increases, the power consumption increases due to the increase in the total FH link power consumption and UE power consumption. In addition, the total system power consumption decreases as the number of the cooperating BSs increases because UEs are free to choose BSs that enable them to transmit with lower transmit power. Furthermore, MCUAA outperforms VNS algorithm mainly due to the utilization of GAP heuristic. With GAP heuristic, the solution is improved within a closed set of minimum power consuming BSs determined for each UE. In VNS, the UEs are assigned to any SBSs in their neighborhood randomly and the solution is improved in a pre-defined number of the changes of neighborhood.

Fig. $2 \mathrm{~b}$ demonstrates the power consumption comparison results obtained for increasing number of the cooperating BSs and SBSs. Increase in the number of the cooperating BSs enables UEs to transmit with less transmit power and provides decrease in total power consumption. Furthermore, MCUAA accomplishes important improvement in comparison to VNS in terms of total power consumption. On the other hand, increase in the number of the SBSs provides decrease in total power consumption since DL and UL association alternatives increase for UEs. As a result, UEs choose the least power consuming BSs for association.

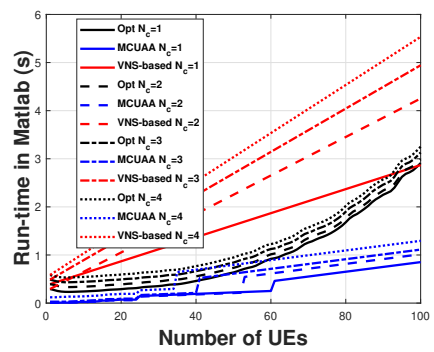

Fig. 3: Effect of number of the UEs on the runtime in Matlab

Fig. 3 illustrates runtime analysis obtained for increasing number of the UEs in Matlab. As the number of the UEs increases, the runtimes of all algorithms increase. However, MCUAA demonstrates significant improvements compared to optimal and VNS-based algorithms since MCUAA is polynomial time and finds the most energy efficient cooperation set of BSs in less time than VNS-based algorithm since it tries to assign UEs only to on BSs rather than trying each BS regardless of whether it is on or off, respectively. Moreover, the runtime increases, as the number of the BSs 


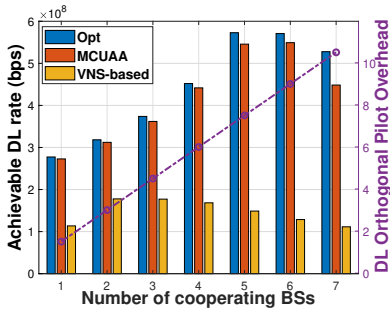

(a) Achievable DL Rate

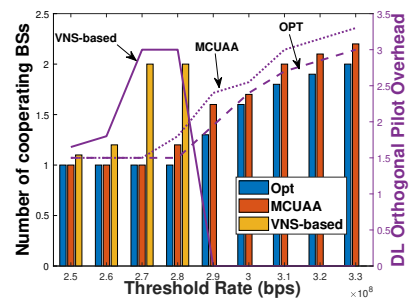

(b) DL Threshold Rate
Fig. 4: number of the Cooperating BSs and Overhead

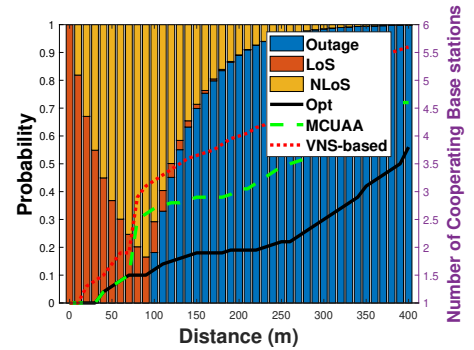

Fig. 5: Distance vs. number of the cooperating BSs

in the cooperation set increases since selection of a larger cooperation set takes more time.

Fig. 4a demonstrates the effect of increase in the number of the cooperating BSs on the achievable rate in the system. As the number of the cooperating BSs increases, achievable rate increases both in DL up to a point. However, it decreases after reaching a specific number of the cooperating BSs due to increase in interference and overhead, in which increasing amount of rate has to be invested into DL orthogonal pilot signaling. Furthermore, the rates achieved by the VNSbased algorithm is less than MCUAA due to selection of cooperating BSs, where the interference inside and outside of the cooperating set is high. Moreover, for the given network architecture with specific number of the UEs, BSs and demanding data rates, 5 cooperating BSs are necessary to get the highest rate.

Fig. $4 \mathrm{~b}$ illustrates the relationship between threshold rate and number of the cooperating BSs. As threshold rate increases, the number of the cooperating BSs increases, which also causes increase in overhead, to fulfill QoS requirements of UEs. However, for increasing threshold rate, the number of the cooperating BSs for VNS-based algorithm becomes zero since the algorithm cannot provide the demanded QoS.

Fig. 5 shows the effect of blockage on UE behavior. As the distance increases, the probability of LoS decreases and probability of NLoS and probability of outage increase. On the other hand, the increase in the distance causes decrease in achievable rate and the number of the cooperating BSs increases to provide the data rate that the UE is demanding. In every condition, MCUAA supports the demanded data rate with less number of the cooperating BSs than VNSbased heuristic since it tries to assign UEs to on BSs rather than turning on additional BSs, which can support the demanded data rate.

\section{CONCLUSION AND Discussion}

In this paper, MC based UL/DL decoupled energy efficient UE association problem in HCRAN is formulated for the first time in the literature. We demonstrate the problem to be NP-hard. We propose polynomial time heuristic algorithm, MCUAA, based on $L P R-R$ and GAP heuristic. We analyze the power consumption, time complexity and achievable rate of MCUAA and verify its superiority compared to existing methods. For future work, our objective is to deepen in MC based UL/DL decoupled energy efficient UE association and consider other metrics such as handover rate and age of information.

\section{REFERENCES}

[1] M. Peng, Y. Li, Z. Zhao and C. Wang, "System architecture and key technologies for $5 \mathrm{G}$ heterogeneous cloud radio access networks," in IEEE Network, vol. 29, no. 2, pp. 6-14, March-April 2015.

[2] A. Ravanshid et al., "Multi-connectivity functional architectures in 5G," 2016 IEEE International Conference on Communications Workshops (ICC), Kuala Lumpur, 2016, pp. 187-192.

[3] D. Aziz et al., "Architecture Approaches for 5G Millimetre Wave Access Assisted by 5G Low-Band Using Multi-Connectivity," 2016 IEEE Globecom Workshops (GC Wkshps), Washington, DC, pp. 1-6.

[4] C. Sheet al., "Improving Network Availability of Ultra-Reliable and Low-Latency Communications With Multi-Connectivity," in IEEE Transactions on Communications, vol. 66, no. 11, pp. 5482-5496,2018.

[5] V. Petrov et al., "Dynamic Multi-Connectivity Performance in UltraDense Urban mmWave Deployments," in IEEE Journal on Selected Areas in Communications, vol. 35, no. 9, pp. 2038-2055, Sept. 2017.

[6] H. Zhang et al., "Energy Efficient User Association and Power Allocation in Millimeter-Wave-Based Ultra Dense Networks With Energy Harvesting Base Stations," in IEEE Journal on Selected Areas in Communications, vol. 35, no. 9, pp. 1936-1947, Sept. 2017.

[7] M. Saimler, S.C. Ergen, "Uplink/downlink decoupled energy efficient user association in heterogeneous cloud radio access networks," in Ad Hoc Networks, vol. 97, Feb. 2020.

[8] K. Zhang et al., "Joint RRH Activation and Robust Coordinated Beamforming for Massive MIMO Heterogeneous Cloud Radio Access Networks," in IEEE Access, vol. 6, pp. 40506-40518, 2018.

[9] L. Wang, W. Yang and M. Pedram, "Towards Green Mobile Networks: Concurrent User and Dynamic Switching in Cells," 2019 IEEE Green Technologies Conference(GreenTech), Lafayette, LA, USA, pp. 1-4.

[10] Lee, G.; Sung, Y.; Seo, J., "Randomly-Directional Beamforming in Millimeter-Wave Multi-User MISO Downlink," in Wireless Communications, IEEE Transactions on , vol.PP, no.99, pp.1-1

[11] C. Ubeda Castellanos et al., "Performance of Uplink Fractional Power Control in UTRAN LTE," VTC Spring 2008 - IEEE Vehicular Technology Conference, Singapore, 2008, pp. 2517-2521.

[12] A. J. Fehske, P. Marsch and G. P. Fettweis, "Bit per Joule efficiency of cooperating base stations in cellular networks," 2010 IEEE Globecom Workshops, Miami, FL, 2010, pp. 1406-1411.

[13] A. Alkhateeb et al., "Limited Feedback Hybrid Precoding for MultiUser Millimeter Wave Systems," in IEEE Transactions on Wireless Communications, vol. 14, no. 11, pp. 6481-6494, Nov. 2015.

[14] M. Deruyck et al., "Reducing the power consumption in Wireless Access Networks: Overview and Recommendations, Progress In Electromagnetics Research, Vol. 132, 255-274, 2012.

[15] R. Ramamonjison, A. Haghnegahdar and V. K. Bhargava, "Joint Optimization of Clustering and Cooperative Beamforming in Green Cognitive Wireless Networks," in IEEE Transactions on Wireless Communications, vol. 13, no. 2, pp. 982-997, February 2014.

[16] K. Smiljkovikj et al., "Efficiency analysis of downlink and uplink decoupling in heterogeneous networks," 2015 IEEE International Conference on Communication Workshop (ICCW), London, pp. 125-130.

[17] S. Miskovic et al., "Variable Neighborhood Search Based Heuristics for the Hard Capacitated K-facility Location Problem," in IPSI BgD Transactions on Internet Research (TIR), vol. 13, no. 1, July 2017.

[18] I.H. Osman, "Heuristics for the Generalised Assignment Problem: Simulated Annealing and Tabu Search Approaches," OR Spektrum, vol. 17 , pp. 211-225, 1995

[19] T. S. Rappaport et al., "Wideband Millimeter-Wave Propagation Measurements and Channel Models for Future Wireless Communication System Design," in IEEE Transactions on Communications, vol. 63, no. 9, pp. 3029-3056, Sept. 2015. 